\title{
British Institute of Radiology: Annual Congress and Exhibition
}

$\mathrm{T}$

THE ninth Annual Congress and Exhibition of the British Institute of Radiology was held in the Central Hall, Westminster, on December 4-6. As usual, one session was devoted to the reading of papers on the physical and technical aspects of $\mathrm{X}$-rays as applied to medicine.

Dr. J. Read, of the Radium Institute, gave a lecture on "High Voltage X-ray Tubes in the U.S.A.", in which he traced the development of continuously evacuated tubes for voltages up to one million volts. The first tube of this type, constructed by Lauritzen at the Kellog Laboratory, Pasadena, in 1927, was successfully operated at $600 \mathrm{k} . \mathrm{v}$. peak supplied by an alternating current generator comprising four transformers in series. The tube, which was self-rectifying, had a glass envelope some nine feet long and an earthed anode. A later tube of the same type has a central earthed discharge-chamber and a balanced arrangement of electrodes, one at $500 \mathrm{k} . \mathrm{v}$. positive potential to earth and the other at $500 \mathrm{k} . \mathrm{v}$. negative potential to earth, so that the peak potential difference between the electrodes is $1000 \mathrm{k} . \mathrm{v}$. Whereas the earlier tube made use of the 'cold emission' of electrons, the later tube has a shielded hot filament. Special vacuum pumps, first of the mercury vapour type with liquid-air traps and later of the oil vapour type, were designed. The latest pump has a pumping speed of 2,000 litres per second.

A somewhat similar X-ray tube excited by alternating voltages up to $100 \mathrm{k} . \mathrm{v}$. peak has been installed at the Soiland Institute. In this tube, the glass envelope is replaced by a porcelain one consisting of a large transformer bushing insulator, this permitting a considerably smaller over-all length. At Lincoln (Illinois) and at the Harper Hospital, Detroit, similar tubes are operated by constant voltage generators. Rather different types of tubes and generators are installed at Seattle and at Chicago. In these installations, the high-tension generators consist of two Villard generators connected in series, and the tubes are built in a cascade arrangement so that the electrons are subjected to successive accelerations. This design reduces the cold emission of electrons, but requires a considerably better vacuum than the Lauritzen design. A further difficulty in operating such tubes arises from the fact that, owing to the long electron path (some 10 feet) the electron beam is deflected by magnetic fields to a serious extent. The effect of the earth's field may be balanced out by suitably disposed magnets, but even so the focus is apt to wander.

Dr. Read also described an ingenious arrangement devised by Sloan at the San Francisco hospital. In this outfit, the high-tension generator consists of an oscillator generating high-frequency waves of 6 metres wave-length, coupled as in a Tesla transformer to a tuned secondary. The transformer is mounted in an evacuated tank, and the high-potential end of the secondary carries the target of the X-ray tube, the filament being mounted in the side of the tank. The high-tension transformer and X-ray tube are thus mounted in the same vessel, and the complete outfit is considerably smaller than those previously considered. Some difficulties are experienced owing to mechanical vibrations of the high-tension coil, and the efficiency of the generator is rather low, an input power of about $100 \mathrm{k} . w$. being required to give a reasonably powerful X-ray beam.

Dr. L. H. Gray, of the Mount Vernon Hospital, gave a paper on "The Ionisation Method of Gamma Ray Energy Measurement", in which he showed theoretically that the degree of ionisation produced in light elements can be related to the energy of the radiation, and measured by ionisation in air. The possibility of measuring gamma ray energies by ionisation depends on two factors : (1) The energy required to produce an ion pair in air must be independent of the velocity of the ionising electrons; and (2), the ratio of the stopping powers of air and other materials for electrons must be independent of the velocity of the electrons. Reviewing the experimental evidence, he is of opinion that both these conditions are fulfilled. In the case of heavy elements, the degree of ionisation is relatively higher, due to the fact that the absorption process is not wholly one of scattering, but is partly photo-electric. In aluminium the ionisation is some 10 per cent higher than in carbon, and in lead more than four times as great.

The eighteenth Silvanus Thompson Memorial Lecture was given by Dr. G. W. C. Kaye, of the National Physical Laboratory, on "Forty Years of Radiology (1895-1935): A Review and Some Reminiscences". Observing that, almost to the day, forty years have passed since Röntgen's discovery of the radiation that bears his name, Dr. Kaye reviewed in a fascinating manner the history of the subject, starting from the early gas X-ray tubes excited by induction coils, passing on to Coolidge's invention of the hot cathode tube (1913), Bouwer's development of the self-protected tube and the recent advances of various types of shock-proof equipment. High-tension generators, he said, beginning as induction coils and made by instrument makers, have become more and more powerful, and modern equipment follows closely the lines of other branches of electrical engineering. Apart from everyday apparatus in use in all hospitals, there is a tendency, particularly in the United States, to experiment with much higher voltages, and generators and tubes operating at one million volts have been described. In Great Britain, X-ray treat. ment with 400 kilovolt $X$-rays is being carried out in several hospitals in London, Leeds, Manchester and Birmingham.

Reviewing the question of dosage, Dr. Kaye stated that the ionisation unit of $\mathrm{X}$-ray quantity, the röntgen, has practically replaced the older arbitrary standards such as the pastille dose. Recent research, he said, appears to show that the röntgen will also serve as a unit of gamma-ray dose. Touching briefly on radium therapy, Dr. Kaye remarked that since 1913 the National Physical Laboratory has measured about a quarter of a pound of radium, of value more than a million pounds, the bulk of this radium being the property of the National Radium Trust. Mention was also made of the industrial and physical applications of X-rays in the radiographic and fluoroscopic examination of objects and the study of the solid state of matter by crystal analysis. 